\title{
External Calibration of Multi-camera System Based on Efficient Pair-Wise Estimation
}

\author{
Chunhui Cui, Wenxian Yang, and King Ngi Ngan \\ Department of Electronic Engineering, \\ The Chinese University of Hong Kong, \\ Hong Kong \\ \{chcui, wxyang, knngan\} @ee.cuhk.edu.hk
}

\begin{abstract}
In this paper, we present an external calibration technique for typical multi-camera system. The technique is very handy in practice using a simple planar pattern. Based on homography, an efficient pair-wise estimation method is proposed to recover the rigid rotation and translation between neighboring cameras. By registering all these partial calibrated structures, complete calibration of the multi-camera system is accomplished. Experiments with both simulated and real data show that accurate and stable calibration results can be achieved by the proposed method.
\end{abstract}

Keywords: Camera calibration, multi-camera system, homography.

\section{Introduction}

Virtual immersive environment usually requires multiple cameras distributed in a wide area, so as to capture scenes of considerable extent in large rooms or even outdoors. A complete multi-camera calibration is an inevitable and important step towards the efficient use of such systems.

In recent years, many multi-camera calibration methods [1][2][3] have been developed based on factorization and global constraints. Usually the whole projection matrix $\mathbf{P}$ is estimated instead of distinguishing intrinsic and extrinsic parameters. The method proposed in [2] relies on the planar pattern and assumes it to be visible to all cameras. Its applications are limited, e.g. unsuitable for wide baseline cases. Other approaches [3] using a laser pointer or virtual calibration object are more flexible, but usually involve elaborate feature detection and tracking, or have some particular requirements in the scene captured.

Some researchers [4][5][6] focus their efforts on the external camera calibration, where the intrinsic and distortion parameters are estimated beforehand and regarded as fixed. In [4], positions and orientations of the model planes relative to the camera are estimated by Zhang's method [7]. Using this information, rigid transforms between two cameras are then determined through an arbitrarily chosen plane. Besides, a RANSAC procedure is applied to remove possible outliers. A more elaborate 
approach is presented in [5], where virtual calibration object is used instead of the planar pattern. A structure-from-motion algorithm is employed to compute the rough pair-wise relationship between cameras. Global registration in a common coordinate system is then performed using a triangulation scheme iteratively. The method proposed in [6] estimates the pair-wise relationship based on the epipolar geometry. Translation and rotation between two cameras are recovered by decomposing the associated essential matrix.

In this paper, we present an external calibration method for typical multi-camera system designed for real-time 3D video acquisition. The technique is simple to use, only requiring the user to present the pattern to cameras in different locations and orientations. Generality in the camera position is offered, only reasonable overlap in FOV (field of view) between neighboring cameras is necessary.

Based on homography, a robust pair-wise estimation method is proposed to recover the rotation and translation between cameras. Four different estimation algorithms are proposed, namely Linear, Two-step, Nonlinear and Three-step method. The four algorithms impose the orthogonal constraint of rotation in different levels and accordingly achieve the calibration results with different accuracy and stability. To calibrate the multi-camera system, the proposed pair-wise calibration method is first applied to estimate the relative relationship between neighboring cameras. Then the complete external calibration is accomplished by registering all these partial calibrated structures. The validity of the proposed method is verified through experiments with both simulated and real data.

\section{Basic Equations from Homography}

Suppose two cameras capture a planar pattern simultaneously as shown in Fig. 1 . Let $\mathbf{m}_{1}=\left[u_{1}, v_{1}\right]^{T}$ and $\mathbf{m}_{2}=\left[u_{2}, v_{2}\right]^{T}$ denote the projections of the same 3D point onto camera 1 and camera 2 , and $\widetilde{\mathbf{m}}_{1}=\left[u_{1}, v_{1}, 1\right]^{T}, \widetilde{\mathbf{m}}_{2}=\left[u_{2}, v_{2}, 1\right]^{T}$ denote their homogeneous coordinates. The homography introduced by the plane is given by

$$
\widetilde{\mathbf{m}}_{2} \simeq \mathbf{H} \widetilde{\mathbf{m}}_{1},
$$

where ' $=$ ' indicates equal up to scale, and $\mathbf{H}$ is the $3 \times 3$ homography matrix.

Let $\mathrm{C} 1$ and $\mathrm{C} 2$ denote the coordinate system of camera 1 and camera 2, respectively, and let $\mathbf{A}_{1}, \mathbf{A}_{2}$ denote their intrinsic matrices. $\mathbf{R}$ and $\mathbf{t}$ represent the rotation and translation from $\mathrm{C} 1$ to $\mathrm{C} 2$ as shown in Fig.1. $\pi$ represents the planar pattern surface with the plane normal $\mathbf{n}=\mathbf{n}_{\pi} / \mathrm{d}_{\pi}$, where $\mathbf{n}_{\pi}$ is the unit vector in the direction of plane normal expressed in $\mathrm{C} 1$, and $\mathrm{d}_{\pi}$ is the distance from the $\mathrm{C} 1$ origin to the plane $\pi$. We then have

$$
\lambda \mathbf{H}=\mathbf{A}_{1}\left(\mathbf{R}+\mathbf{t} \cdot \mathbf{n}^{T}\right) \mathbf{A}_{2},
$$

where $\lambda$ is an unknown arbitrary scalar. 


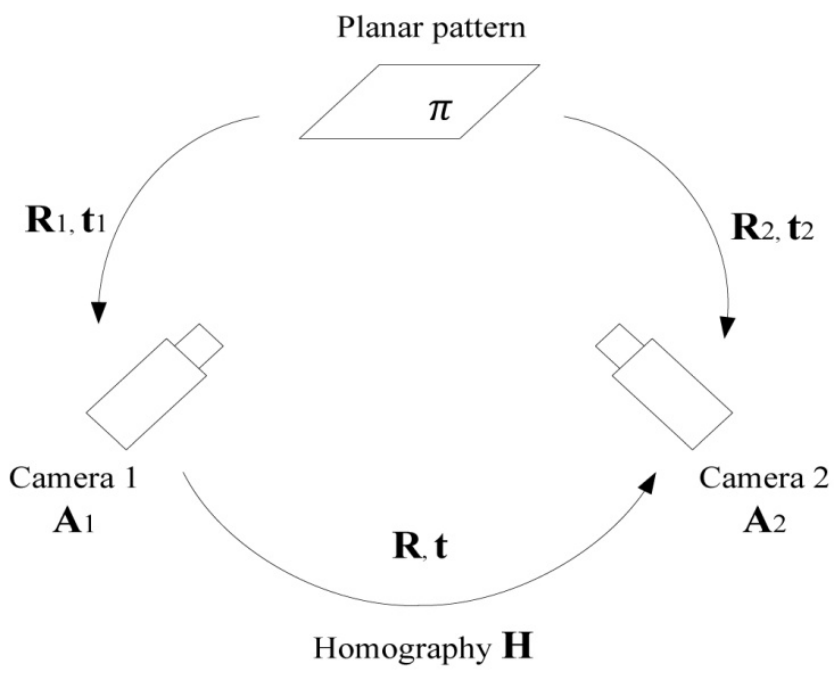

Fig. 1. Homography between two views

\section{Multi-camera Calibration Based on Pair-Wise Estimation}

In most multiview applications, the intrinsic and distortion parameters of cameras are fixed. Therefore it is reasonable to estimate these parameters for each camera independently and elaborately, so as to achieve accurate and stable calibration. We apply Zhang's method [7] to do the intrinsic calibration for each individual camera. Thus only the external calibration is necessary every time the cameras are moved and refocused to capture new 3D video.

Planar pattern such as a checkerboard is widely used in calibration due to its flexibility and convenience. The main drawback of using the planar pattern lies in its invisibility to all cameras. However, we only use the planar pattern to estimate the relative relationship between two neighboring cameras. It is practical to make the pattern visible to both cameras, because in most multiview systems two neighboring cameras generally have sufficient common FOV. As for global registration, the transform from one camera to another can be easily computed by chaining those associated neighboring transforms together. It is argued that the chaining procedure is prone to errors. However, accurate and stable pair-wise calibration can benefit the accuracy of chaining. Our experiments show that there is no obvious error accumulation during transform chaining using the proposed pair-wise estimation method.

An easy way to do the pair-wise $(\mathbf{R}, \mathbf{t})$ estimation is to utilize the single camera calibration results. Intrinsic calibration [7] can also recover the positions and orientations of the model planes relative to the camera, such as $\left(\mathbf{R}_{1}, \mathbf{t}_{1}\right)$ and $\left(\mathbf{R}_{2}, \mathbf{t}_{2}\right)$ in Fig. 1 . Using this information, $(\mathbf{R}, \mathbf{t})$ between two cameras can be determined through an 
arbitrarily chosen model plane. Ideally, $(\mathbf{R}, \mathbf{t})$ between cameras should be invariant irrespective of the plane through which they are computed. However in the presence of noise, the $(\mathbf{R}, \mathbf{t})$ estimates computed through different planes actually differ from each other. Simply combining these estimates is not robust and may lead to erroneous or unstable calibration results.

Based on homography (2), we propose a robust pair-wise estimation method to recover the relative relationship between two cameras. First, homography $\mathbf{H}$ is estimated by correspondence, and then follows the calculation of unknown scale $\lambda$ and plane normal $\mathbf{n}$. Finally, $(\mathbf{R}, \mathbf{t})$ between two cameras can be estimated by four different algorithms: Linear, Two-step, Nonlinear and Three-step method.

\subsection{Homography Estimation}

With sufficient point correspondences, the homography matrix $\mathbf{H}$ can be computed based on (1). The algorithm described in [7] is applied to do the homography estimation. As shown in Fig. 1, each image pair, one view from camera 1 and the other from camera 2, leads to a homography $\mathbf{H}$. Suppose there are total $P$ image pairs and then we can estimate $P$ homographies $\mathbf{H}_{i}(i=1,2, \ldots, P)$ induced by different planes.

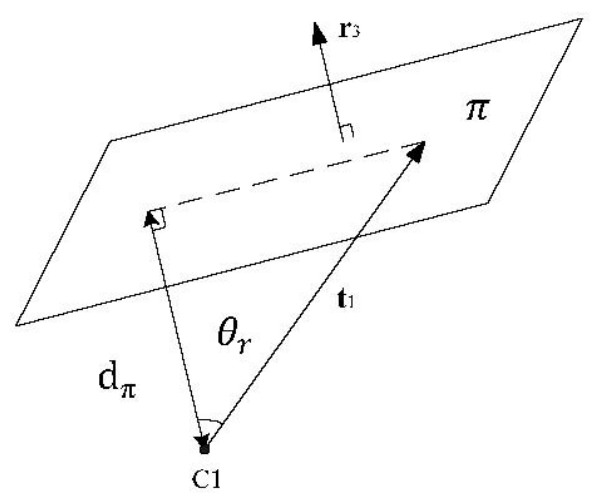

Fig. 2. Geometry between the model plane and camera center

\subsection{Calculation of $\mathbf{n}$ and $\lambda$}

The plane normal $\mathbf{n}$ also varies with the moving pattern, thus $P$ different stereo views lead to $P$ different normals $\mathbf{n}_{i}(i=1,2, \ldots, P)$. To compute each plane normal w.r.t. the $\mathrm{C} 1$ coordinates system, we first use Zhang's method [7] to estimate the plane position and orientation relative to C1, i.e. $\left(\mathbf{R}_{1}, \mathbf{t}_{1}\right)$.

Let's express $\mathbf{R}_{1}$ by means of its column vectors $\mathbf{R}_{1}=\left[\mathbf{r}_{1} \mathbf{r}_{2} \mathbf{r}_{3}\right]$. As shown in Fig. 2, the third column vector $\mathbf{r}_{3}$ is a unit vector parallel to the plane normal, therefore $\mathbf{r}_{3}=\mathbf{n}_{\pi}$. The translation $\mathbf{t}_{1}$ is a vector connecting the $\mathrm{C} 1$ origin to a specific point on the plane $\pi$. Since $\mathbf{r}_{3}$ is orthogonal to the plane $\pi$, the distance from C1 origin to the plane can be computed by 


$$
\mathrm{d}_{\pi}=\left|\mathbf{t}_{1}\right| \cos \theta_{r t}=\left|\mathbf{r}_{3}\right|\left|\mathbf{t}_{1}\right| \cos \theta_{r t}=\mathbf{r}_{3} \cdot \mathbf{t}_{1},
$$

where $\theta_{r t}$ denotes the angle between $\mathbf{r}_{3}$ and $\mathbf{t}_{1}$.

Therefore the plane normal $\mathbf{n}$ can be calculated by $\mathbf{r}_{3}$ and $\mathbf{t}_{1}$ as

$$
\mathbf{n}=\frac{\mathbf{n}_{\pi}}{\mathrm{d}_{\pi}}=\frac{\mathbf{r}_{3}}{\mathbf{r}_{3} \cdot \mathbf{t}_{1}}
$$

The collineation matrix $\left(\mathbf{R}+\mathbf{t} \cdot \mathbf{n}^{T}\right)$ has an important property that its median singular value is equal to one [8]. This property can be used to compute the unknown scalar $\lambda$. Let's define $\mathbf{G}=\lambda \mathbf{A}_{1}^{-1} \mathbf{H} \mathbf{A}_{2}^{-1}$, follow Eqn. (2) we have

$$
\mathbf{G}=\mathbf{R}+\mathbf{t} \cdot \mathbf{n}^{T}
$$

Let $\left(\sigma_{1}, \sigma_{2}, \sigma_{3}\right)$ denote the singular values of matrix $\mathbf{A}_{1}^{-1} \mathbf{H} \mathbf{A}_{2}^{-1}$ in descending order $\left(\sigma_{1} \geq \sigma_{2} \geq \sigma_{3}>0\right)$. According to (5), matrix $\mathbf{G}=\lambda \mathbf{A}_{1}^{-1} \mathbf{H} \mathbf{A}_{2}^{-1}$ has median singular value equal to one, thus we have

$$
\lambda \sigma_{2}=1
$$

Note that matrix $\mathbf{A}_{1}, \mathbf{A}_{2}$ and $\mathbf{H}$ are known, so we can compute $\lambda$ according to (6) and then recover the matrix $\mathbf{G}$.

\section{3 (R, t) Estimation}

\subsubsection{Linear Method}

From Eqn. (5), we can derive the following linear equation (7), where vec(X) denotes the vectorization of matrix $\mathbf{X}$ formed by stacking the columns of $\mathbf{X}$ into a single column vector, $\mathbf{I}_{3}$ and $\mathbf{I}_{9}$ denote $3 \times 3$ and $9 \times 9$ identity matrices, respectively, and $\otimes$ denotes the kronecker product.

$$
\underbrace{\left[\begin{array}{ll}
\mathbf{I}_{9} & \mathbf{n} \otimes \mathbf{I}_{3}
\end{array}\right]}_{9 \times 12} \underbrace{\left[\begin{array}{c}
\operatorname{vec}(\mathbf{R}) \\
\mathbf{t}
\end{array}\right]}_{12 \times 1}=\underbrace{\operatorname{vec}(\mathbf{G})}_{9 \times 1}
$$

As described earlier, each stereo view of the pattern can generate a homography $\mathbf{H}_{i}$ and a normal $\mathbf{n}_{i}$, and from each $\mathbf{H}_{i}$ the normalized matrix $\mathbf{G}_{i}$ can then be recovered. As a set of $\mathbf{G}_{i}$ and $\mathbf{n}_{i}$ form an equation (7), by stacking $P$ equations we have

$$
\underbrace{\left[\begin{array}{cc}
\mathbf{I}_{9} & \mathbf{n}_{1} \otimes \mathbf{I}_{3} \\
& \vdots \\
\mathbf{I}_{9} & \mathbf{n}_{P} \otimes \mathbf{I}_{3}
\end{array}\right]}_{9 P \times 12} \underbrace{\left[\begin{array}{c}
\operatorname{vec}(\mathbf{R}) \\
\mathbf{t}
\end{array}\right]}_{12 \times 1}=\underbrace{\left[\begin{array}{c}
\operatorname{vec}\left(\mathbf{G}_{1}\right) \\
\vdots \\
\operatorname{vec}\left(\mathbf{G}_{P}\right)
\end{array}\right]}_{9 P \times 1}
$$

Let $\mathbf{D}$ denote the left $9 P \times 12$ matrix and $\mathbf{d}$ denote the right $9 P \times 1$ matrix. The leastsquares solution to (8) is given by 


$$
\left[\begin{array}{c}
\operatorname{vec}(\mathbf{R}) \\
\mathbf{t}
\end{array}\right]=\left(\mathbf{D}^{T} \mathbf{D}\right)^{-1} \mathbf{D}^{T} \mathbf{d}
$$

Because of noise in data, the computed matrix $\mathbf{R}$ does not in general satisfy the orthogonal property of a rotation matrix. We need to solve the best orthogonal matrix $\mathbf{R}^{\prime}$ to approximate $\mathbf{R}$. The method described in [7] is adopted here.

However, the orthogonal approximation causes a severe problem here. $(\mathbf{R}, \mathbf{t})$ computed by (9) is the best solution to equation (8) in the least square sense. After orthogonal approximation, the obtained $\left(\mathbf{R}^{\prime}, \mathbf{t}\right)$ no longer fit this equation well and may lead to erroneous calibration results. Therefore it is necessary to impose the orthogonal constraint $\mathbf{R}^{T} \mathbf{R}=\mathbf{I}$ in the $(\mathbf{R}, \mathbf{t})$ estimation procedure so that the matrix $\mathbf{R}$ is as close to orthogonal as possible, consequently less deviation will be caused by orthogonal approximation from $\mathbf{R}$ to $\mathbf{R}^{\prime}$.

\subsubsection{Two-Step Method with Implicit Orthogonal Constraint}

In this section, we first derive an implicit constraint imposed in vector $\mathbf{t}$ based on homography and the orthogonal property of matrix $\mathbf{R}$. Then a Two-step method is proposed to estimate the pair-wise $(\mathbf{R}, \mathbf{t})$, where the implicit orthogonal constraint is imposed leading to better calibration results compared with the linear method.

\section{Implicit Orthogonal Constraint}

Follow Eqn. (5) we have

$$
\mathbf{R}=\mathbf{G}-\mathbf{t} \cdot \mathbf{n}^{\mathbf{T}}=\left[\begin{array}{l}
\mathbf{g}_{1}^{T}-t_{1} \mathbf{n}^{T} \\
\mathbf{g}_{2}^{T}-t_{2} \mathbf{n}^{T} \\
\mathbf{g}_{3}^{T}-t_{3} \mathbf{n}^{T}
\end{array}\right]
$$

where $\mathbf{t}=\left[\begin{array}{c}t_{1} \\ t_{2} \\ t_{3}\end{array}\right], \mathbf{G}=\left[\begin{array}{l}\mathbf{g}_{1}^{T} \\ \mathbf{g}_{2}^{T} \\ \mathbf{g}_{3}^{T}\end{array}\right]$ and $\mathbf{g}_{i}^{T}(i=1,2,3)$ denote the three row vectors of $\mathbf{G}$.

As matrix $\mathbf{R}$ is orthogonal, the three row vectors $\mathbf{g}_{i}^{T}-t_{i} \mathbf{n}^{T}(i=1,2,3)$ form an orthonormal basis of $\mathbf{R}^{\mathbf{3}}$, i.e., we have

$$
\left\{\begin{array}{l}
\left(\mathbf{g}_{i}^{T}-t_{i} \mathbf{n}^{T}\right)\left(\mathbf{g}_{i}-t_{i} \mathbf{n}\right)=1 \\
\left(\mathbf{g}_{j}^{T}-t_{j} \mathbf{n}^{T}\right)\left(\mathbf{g}_{j}-t_{j} \mathbf{n}\right)=1 \\
\left(\mathbf{g}_{i}^{T}-t_{i} \mathbf{n}^{T}\right)\left(\mathbf{g}_{j}-t_{j} \mathbf{n}\right)=0
\end{array} \quad(i, j \in[1,2,3] ; i \neq j)\right.
$$

Note that $\mathbf{g}_{i}^{T} \mathbf{n}=\mathbf{n}^{T} \mathbf{g}_{i}(i=1,2,3)$, we then have

$$
\left\{\begin{array}{c}
\mathbf{g}_{i}^{T} \mathbf{g}_{i}+t_{i}^{2} \mathbf{n}^{T} \mathbf{n}-1=2 t_{i} \mathbf{n}^{T} \mathbf{g}_{i} \\
\mathbf{g}_{j}^{T} \mathbf{g}_{j}+t_{j}^{2} \mathbf{n}^{T} \mathbf{n}-1=2 t_{j} \mathbf{n}^{T} \mathbf{g}_{j} \\
\mathbf{g}_{i}^{T} \mathbf{g}_{j}+t_{i} t_{j} \mathbf{n}^{T} \mathbf{n}=t_{i} \mathbf{n}^{T} \mathbf{g}_{j}+t_{j} \mathbf{n}^{T} \mathbf{g}_{i}
\end{array} \quad(i, j \in[1,2,3] ; i \neq j)\right.
$$


By eliminating the terms involving $\mathbf{n}$, we can derive the quadratic equation (13) with one unknown quantity $\mathrm{k}_{i j}=\mathrm{t}_{i} / \mathrm{t}_{j}$. Note that Eqn. (13) no longer involves the normal $\mathbf{n}$, indicating less noise disturbance.

$$
2 \mathbf{g}_{i}^{T} \mathbf{g}_{j}=\frac{t_{i}}{t_{j}}\left(\mathbf{g}_{j}^{T} \mathbf{g}_{j}-1\right)+\frac{t_{j}}{t_{i}}\left(\mathbf{g}_{i}^{T} \mathbf{g}_{i}-1\right) \quad(i, j \in[1,2,3] ; i \neq j)
$$

\section{Two-Step Method}

The proposed Two-step method is based on the implicit orthogonal constraint derived above. At the first step, we gather $P$ such equations as (13) corresponding to different $\mathbf{G}$ matrices and compose simultaneous quadratic equations. Solving this problem by least square metric, we can obtain the uniform ratio of the three elements of $\mathbf{t}$ vector $t_{1}: t_{2}: t_{3}=1: k_{21}: k_{31}$. Therefore, the original 3-DOF (Degree of Freedom) $\mathbf{t}$ vector is reduced to a single scale $s$ as

$$
\mathbf{t}=s\left[\begin{array}{c}
1 \\
k_{21} \\
k_{31}
\end{array}\right]
$$

Based on (14), we rewrite Eqn. (7) as (15). At the second step, we solve the simultaneous linear equations generated by stacking $P$ such equations as (15). Once $s$ is estimated, vector $\mathbf{t}$ is readily computed by (14).

$$
\underbrace{\operatorname{vec}(\mathbf{G})}_{9 \times 1}=\operatorname{vec}(\mathbf{R})+\mathbf{n} \otimes\left[\begin{array}{c}
1 \\
k_{21} \\
k_{31}
\end{array}\right] s=\underbrace{\left[\mathbf{I}_{9} \mathbf{n} \otimes\left[\begin{array}{c}
1 \\
k_{21} \\
k_{31}
\end{array}\right]\right.}_{9 \times 10}] \underbrace{\left[\begin{array}{c}
\operatorname{vec}(\mathbf{R}) \\
s
\end{array}\right]}_{10 \times 1}
$$

The Two-step method imposes the implicit orthogonal constraint (13) in the estimation explicitly, while keeps the problem linear. $(\mathbf{R}, \mathbf{t})$ estimated by this method not only conform to the homography geometry, but also satisfy the orthogonal constraint $\mathbf{R}^{T} \mathbf{R}=\mathbf{I}$ better. Still $\mathbf{R}$ is not perfectly orthogonal and further orthogonal approximation is necessary. However, less deviation will be induced by the approximation, because $\mathbf{R}$ is much closer to its corresponding orthogonal approximation $\mathbf{R}^{\prime}$.

\subsubsection{Nonlinear Method}

If we expect the estimated matrix $\mathbf{R}$ to be orthogonal without further orthogonal approximation, we should impose the constraint $\mathbf{R}^{T} \mathbf{R}=\mathbf{I}$ explicitly in the estimation and it turns to be a constrained nonlinear optimization problem:

$$
\min _{\mathrm{R}, \mathrm{t}} \sum_{i}\left\|\mathbf{G}_{i}-\mathbf{R}-\mathbf{t} \cdot \mathbf{n}_{i}^{T}\right\|_{\mathrm{F}} \quad \text { subject to } \mathbf{R}^{T} \mathbf{R}=\mathbf{I},
$$

where the optimum is in the sense of the smallest sum of Frobenius norms.

We may use the Lagrange Multiplier to solve the constrained problem, but a better choice is to utilize the angle-axis representation of rotation. As we know, in three dimensions a rotation can be defined by a single angle of rotation $\theta$, and the direction 
of a unit vector $\mathbf{v}=(x, y, z)$, about which to rotate. Thus the rotation matrix $\mathbf{R}$ can be written as

$$
\mathbf{R}(\mathbf{v}, \theta)=\left[\begin{array}{ccc}
\cos \theta+(1-\cos \theta) x^{2} & (1-\cos \theta) x y-(\sin \theta) z & (1-\cos \theta) x z+(\sin \theta) y \\
(1-\cos \theta) x y+(\sin \theta) z & \cos \theta+(1-\cos \theta) y^{2} & (1-\cos \theta) y z-(\sin \theta) x \\
(1-\cos \theta) x z-(\sin \theta) y & (1-\cos \theta) y z+(\sin \theta) x & \cos \theta+(1-\cos \theta) z^{2}
\end{array}\right]
$$

Then we substitute this compact representation to the minimization term of (16), and solve the nonlinear problem with the Levenberg-Marquardt algorithm as implemented in Minpack [9]. The required initial guess of $(\mathbf{R}, \mathbf{t})$ can be obtained either by the linear method or by the Two-step method. Experiment results show that matrix $\mathbf{R}$ estimated by the nonlinear method is already orthogonal, thus further orthogonal approximation is unnecessary, consequently avoiding the problem caused by it.

\subsubsection{Three-Step Method}

Although the nonlinear method is expected to achieve the best result, it is indeed time-consuming. Another choice is to make a reasonable trade-off, i.e., to develop a method that has much less computational complexity and meantime can achieve comparable performance. By good calibration result, we mean that the $(\mathbf{R}, \mathbf{t})$ should not only conform to the homography geometry, but also satisfy the orthogonal straint $\mathbf{R}^{T} \mathbf{R}=\mathbf{I}$. Though we cannot obtain this kind of result completely by a linear method, it is possible to achieve a $\operatorname{good}(\mathbf{R}, \mathbf{t})$ estimation with close performance through several linear optimization steps.

As mentioned before, a rotation matrix in three dimensions can be represented by a unit vector $\mathbf{v}=(x, y, z)$ and an angle $\theta$. According to Euler's rotation theorem, the $3 \times 3$ rotation matrix $\mathbf{R}$ has one real eigenvalue equal to unity, and the unit vector $\mathbf{v}$ is the corresponding eigenvector, i.e.

$$
\mathbf{R v}=\mathbf{v}
$$

It follows from (5) and (18) that

$$
\mathbf{G v}=\mathbf{v}+\mathbf{t} \cdot \mathbf{n}^{T} \cdot \mathbf{v}
$$

If we know the matrix $\mathbf{G}_{i}, \mathbf{n}_{i}(i=1, \ldots, P)$ and $\mathbf{t}$, vector $\mathbf{v}$ can be estimated by solving the linear equation (20), which is the accumulation of $P$ such equations as (19).

$$
\left[\begin{array}{c}
\mathbf{G}_{1}-\mathbf{I}-\mathbf{t} \cdot \mathbf{n}_{1}^{T} \\
\vdots \\
\mathbf{G}_{P}-\mathbf{I}-\mathbf{t} \cdot \mathbf{n}_{P}^{T}
\end{array}\right] \mathbf{v}=\mathbf{0}
$$

According to Eckart-Young-Mirsky (EYM) theorem, the solution to Eqn. (20), in matrix form as $\mathbf{B v}=\mathbf{0}$, is the right singular vector of $\mathbf{B}$ associated with its smallest singular value. 
To recover the rotation matrix, we further estimate the parameter $\theta$ based on the rotation representation (21), where $\hat{\mathbf{v}}$ indicates the $3 \times 3$ skew symmetric matrix corresponding to $\mathbf{v}$.

$$
\mathbf{R}=\mathbf{I}+(\sin \theta) \hat{\mathbf{v}}+(1-\cos \theta) \hat{\mathbf{v}}^{2}
$$

In order to guarantee a linear optimization, we estimate two parameters $\cos \theta$ and $\sin \theta$ instead of the single $\theta$ and the constraint $\cos ^{2} \theta+\sin ^{2} \theta=1$ is not imposed in the estimation. Experimental results show that the $\operatorname{computed} \cos \theta$ and $\sin \theta$ basically satisfy this constraint.

At this step, we may retain the original result of vector $\mathbf{t}$, or we can refine it together with $\cos \theta$ and $\sin \theta$, while still keep the optimization problem a linear one. In order to achieve robust results, we choose the latter scheme to estimate $\cos \theta, \sin \theta$ and vector $\mathbf{t}$ together by linear equation (22), which is derived from (5) and (21)

$$
\left[\operatorname{vec}(\hat{\mathbf{v}})-\operatorname{vec}\left(\hat{\mathbf{v}}^{2}\right) \quad \mathbf{n} \otimes \mathbf{I}\right]\left[\begin{array}{c}
\sin \theta \\
\cos \theta \\
\mathbf{t}
\end{array}\right]=\operatorname{vec}\left(\mathbf{G}-\mathbf{I}-\hat{\mathbf{v}}^{2}\right)
$$

By stacking $P$ such equations, we have

$$
\underbrace{\left[\begin{array}{ccc}
\operatorname{vec}(\hat{\mathbf{v}}) & -\operatorname{vec}\left(\hat{\mathbf{v}}^{2}\right) & \mathbf{n}_{1} \otimes \mathbf{I} \\
\vdots & \vdots & \vdots \\
\operatorname{vec}(\hat{\mathbf{v}}) & -\operatorname{vec}\left(\hat{\mathbf{v}}^{2}\right) & \mathbf{n}_{P} \otimes \mathbf{I}
\end{array}\right]}_{9 P \times 5}[\underbrace{\left[\begin{array}{c}
\sin \theta \\
\cos \theta \\
\mathbf{t}
\end{array}\right]}_{5 \times 1}=\underbrace{\left[\begin{array}{c}
\operatorname{vec}\left(\mathbf{G}_{1}-\mathbf{I}-\hat{\mathbf{v}}^{2}\right) \\
\vdots \\
\operatorname{vec}\left(\mathbf{G}_{P}-\mathbf{I}-\hat{\mathbf{v}}^{2}\right)
\end{array}\right]}_{9 P \times 1}
$$

Based on the above description, the Three-step method is outlined as follows:

1. Use linear method or Two-step method to compute the initial estimation of $\mathbf{t}$;

2. Estimate vector $\mathbf{v}$ based on (20) with $\mathbf{t}$ fixed;

3. Estimate parameters $\cos \theta, \sin \theta$ and refine $\mathbf{t}$ together based on (23) with $\mathbf{v}$ fixed. With $\cos \theta, \sin \theta$ and $\mathbf{v}$ already known, the rotation matrix $\mathbf{R}$ can be recovered by (21).

\section{Experimental Results}

\subsection{MPLD}

The mean point-line distance (MPLD) is used as the metric to evaluate the extrinsic calibration results. Suppose two cameras capture the same scene from different views, and a pair of images $\mathbf{I}_{1}$ and $\mathbf{I}_{2}$ is obtained. If the two cameras are calibrated both intrinsically and extrinsically, the associated fundamental matrix $\mathbf{F}$ can be recovered by (24).

$$
\mathbf{F}=\mathbf{A}_{2}^{-T} \mathbf{R}[\mathbf{t}]_{\mathrm{X}} \mathbf{A}_{1}^{-1}
$$


With $\mathbf{F}$ known, given a point $\mathbf{m}_{1}$ in $\mathbf{I}_{1}$, we can determine the corresponding epipolarline $\boldsymbol{l}_{1}$ in $\mathbf{I}_{2}$. Ideally, the correspondence of $\mathbf{m}_{1}$ in $\mathbf{I}_{2}$, i.e. $\mathbf{m}_{2}$ should be located on the epipolar-line $\mathbf{l}_{1}$. However, it may deviate from $\boldsymbol{l}_{1}$ due to data noise or inaccurate calibration. Therefore the distance of $\mathbf{m}_{2}$ to $\boldsymbol{l}_{1}$ can be used reasonably to evaluate the calibration performance. The mean point line distance is computed over all the pattern corners of all the test images.

\subsection{Simulated Data}

This simulation is to evaluate the performance of different $(\mathbf{R}, \mathbf{t})$ estimation algorithms, especially their robustness to data noise. We consider the scenario that two cameras capture a planar pattern with $9 \times 12$ corners. Total 20 different stereo views of the plane are used. We specify the translations and rotations from the plane to the first camera and from the first camera to the second one. Gaussian noise is added to the true projected image points (we assume no distortion), from a standard deviation of 0.05 pixels up to 0.45 pixels in step of 0.05 . Fig. 3 shows the mean of MPLD from 50 simulations by different algorithms. We also give the simulation results of Zhang's method [7] for comparison. As Zhang's method can estimate the transformation between two cameras from a single stereo image pair, in our experiment we simply take the mean of the $(\mathbf{R}, \mathbf{t})$ estimates from different image pairs and denote this method as Zhang_mean. For both experiments here and those in Section 4.3, the required $\mathbf{t}$ for the Three-step method is initialized by the Two-step method.

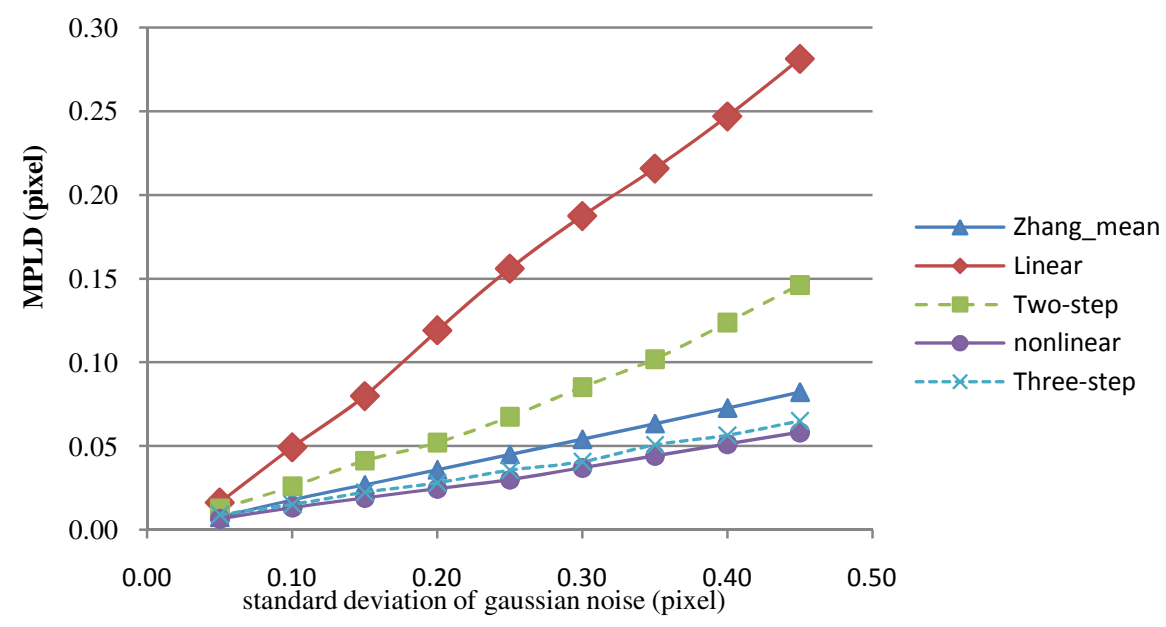

Fig. 3. Simulation results of different methods

As shown in Fig.3, the performance of the original linear method is bad mainly due to the orthogonal approximation problem. With implicit orthogonal constraint imposed, the Two-step method performs much better although still not satisfactory. As 
expected, the nonlinear method achieves the best results in the sense of robustness against data noise. The Three-step method has very close performance and meantime much lower complexity. By our rough test on the PC with $1.83 \mathrm{G}$ Duo CPU and $1.5 \mathrm{G}$ RAM, the nonlinear $(\mathbf{R}, \mathbf{t})$ estimation procedure may take dozens of seconds, while by all the other algorithms the time-consuming keeps within $10 \mathrm{~ms}$. Both the nonlinear and Three-step methods are less sensitive to noise compared with Zhang_mean.

\subsection{Real Data}

Experiments are also performed using real data captured by our multi-camera system that consists of five Prosilica GC650C cameras with $8 \mathrm{~mm}$ lens. The cameras are indexed from 0 to 4 sequentially, thus there are total four neighboring pairs: $(0-1),(1-2)$, (2-3) and (3-4). In the experiment, the five cameras are placed focusing on the same scene. The angle between the optical axes of two neighboring cameras is approximately 30 degree. Beforehand, intrinsic calibration was done to the five cameras individually using Zhang's method [7]. The proposed method is then applied to recover the extrinsic relationship among cameras. A checkerboard pattern with $9 \times 12$ corners is presented to the four camera pairs. For each pair, 30 different stereo views are captured. To verify the calibration results, another checkerboard with $6 \times 9$ corners is used and similarly 30 views are captured for each camera pair. Calibration is performed by different $(\mathbf{R}, \mathbf{t})$ estimation algorithms, while using the same training and testing images. As shown in Table 1, the experimental results of real data are similar to that of synthesized data. Both the nonlinear and Three-step method outperform the method Zhang_mean. Note that for all methods here, we do not apply any processing to remove possible data outliers. Better results can be expected by adopting such procedure.

Table 1. Results of pair-wise $(\mathbf{R}, \mathbf{t})$ estimation

\begin{tabular}{|c|c|c|c|c|c|}
\hline \multirow{2}{*}{$\begin{array}{c}\text { Camera } \\
\text { Pairs }\end{array}$} & \multicolumn{5}{|c|}{ MPLD (pixel) by different methods } \\
\cline { 2 - 6 } & Zhang_mean & Linear & Two-step & Nonlinear & Three-step \\
\hline $0-1$ & 0.0825 & 0.5297 & 0.0851 & 0.0781 & 0.0799 \\
\hline $1-2$ & 0.0814 & 0.3526 & 0.2400 & 0.0655 & 0.0617 \\
\hline $2-3$ & 0.0692 & 0.3359 & 0.0610 & 0.0488 & 0.0540 \\
\hline $3-4$ & 0.0769 & 0.4112 & 0.1505 & 0.0654 & 0.0693 \\
\hline Average & 0.0775 & 0.4074 & 0.1342 & 0.0644 & 0.0662 \\
\hline
\end{tabular}

To investigate the error induced by transform chaining, we test the calibration results of camera pairs: $(0-2),(0-3)$ and $(0-4)$. $(\mathbf{R}, \mathbf{t})$ between these camera pairs are computed by chaining the $(\mathbf{R}, \mathbf{t})$ results across those neighboring pairs (0-1), (1-2), (2-3) and (3-4). As shown in Fig. 4, there is no obvious error accumulation during the $(\mathbf{R}, \mathbf{t})$ chaining procedure, especially when the pair-wise calibration results are sufficiently accurate and stable, as estimated by the Three-step or nonlinear method. Clearly the calibration results can be further improved by using more robust registration method such as [6], rather than simple chaining here. 


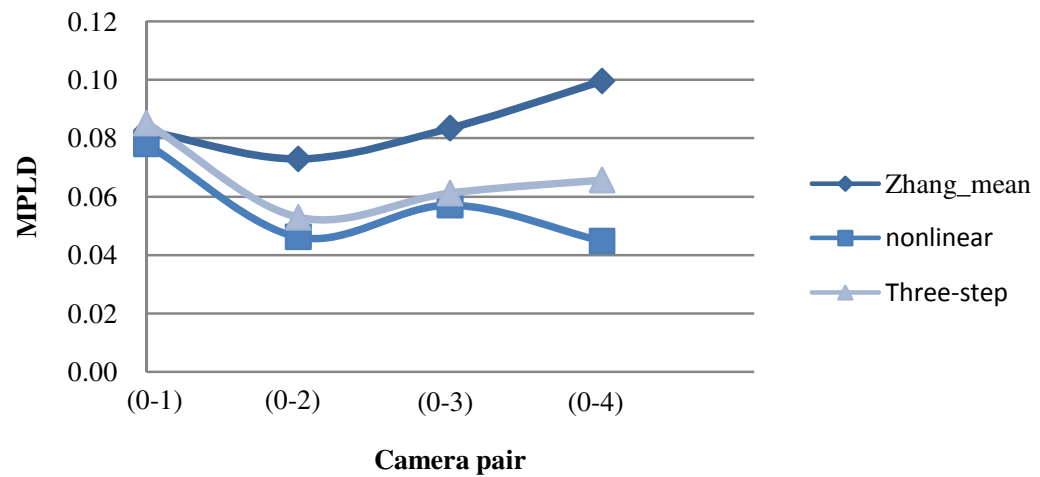

Fig. 4. Results of transform chaining

\section{Conclusion}

In this paper, we present a convenient and efficient method to calibrate the typical multi-camera system. Relative relationship between neighboring cameras is first recovered by the proposed pair-wise estimation method. Complete multi-camera calibration is then accomplished by chaining these pair-wise estimates together. Four estimation algorithms are proposed based on homography. The linear method does not impose any constraint, thus leads to inaccurate calibration results due to the problem caused by orthogonal approximation. Better results are obtained by the Two-step method with the implicit orthogonal constraint imposed. The orthogonal constraint is fully imposed in the nonlinear method, which achieves the best calibration results in the sense of noise robustness. The Three-step method has very close performance to the nonlinear method, while has much lower computational complexity. Extra outlier removal and more robust registration method may further improve the accuracy and stability of the external calibration.

\section{References}

1. Sturm, P., Triggs, B.: A Factorization Based Algorithm for Multi-Image Projective Structure and Motion. In: European Conference on Computer Vision, pp. 709-720 (1996)

2. Ueshiba, T., Tomita, F.: Plane-based Calibration Algorithm for Multi-camera Systems via Factorization of Homography Matrices. In: International Conference on Computer Vision, vol. 2, pp. 966-973 (2003)

3. Svoboda, T., Martinec, D., Pajdla, T.: A Convenient Multicamera Self-calibration for Virtual Environments. PRESENCE: Teleoperators and Virtual Environments 14(4) (2005)

4. Prince, S., Cheok, A.D., Farbiz, F., Williamson, T., Johnson, N., Billinghurst, M., Kato, H.: Nat: 3D Live: Real Time Captured Content for Mixed Reality. In: International Symposium on Mixed and Augmented Reality, pp. 307-317 (2002)

5. Chen, X., Davis, J., Slusallek, P.: Wide Area Camera Calibration Using Virtual Calibration Objects. Computer Vision and Pattern Recognition 2, 520-527 (2000)

6. Hrke, I., Ahrenberg, L., Magnor, M.: External Camera Calibration for Synchronized Multivideo Systems. Journal of WSCG 12 (2004) 
7. Zhang, Z.Y.: A Flexible New Technique for Camera Calibration. IEEE Transactions on Pattern Analysis and Machine Intelligence 22(11), 1330-1334 (2000)

8. Zhang, Z., Hanson, A.R.: Scaled Euclidean 3D Reconstruction Based on Externally Uncalibrated Cameras. In: IEEE Symposium on Computer Vision, pp. 37-42 (1995)

9. More, J.: The Levenberg-marquardt Algorithm, Implementation and Theory. In: Watson, G.A. (ed.) Numerical Analysis. Lecture Notes in Mathematics, vol. 630, Springer, Heidelberg (1977) 